\title{
Monitoring the Earth's Dynamic Magnetic Field
}

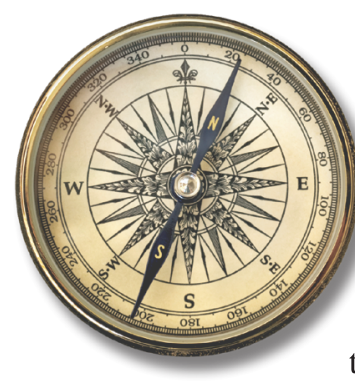

Gor centuries, the compass has been used for orientation and navigation, with the north-seeking tendency of its magnetized needle responding to the Earth's magnetic field. The main part of the field originates in the Earth's deep interior, but the field also extends outwards into space. The field is complicated in shape and it changes over time. As a result, magnetic maps and charts need to be updated every few years, an on-going project that requires the collection of magnetic data from satellites, dedicated surveys, and permanent observatories located around the world. In recent years, monitoring of the Earth's magnetic field has become important for mitigating the impacts of space weather. Magnetic storms, or periods of time when the field is unusually active, are caused by the dynamic

interaction of

the Earth's magnetic field with the Sun (fig. 1). It is during magnetic storms that beautiful auroral lights can be seen at high latitudes.

But magnetic storms can also adversely affect the infrastructure and activities of our modern, technologically based society. Large storms can cause the loss of radio communication, reduce the accuracy of global-positioning systems, damage satellite electronics and affect satellite operations, enhance radiation levels for astronaut and high-altitude pilots, increase pipeline corrosion, and induce voltage surges in electric power grids, causing blackouts. Consequently, the science of geomagnetism is more important today than at any point in the discipline's long and colorful history.

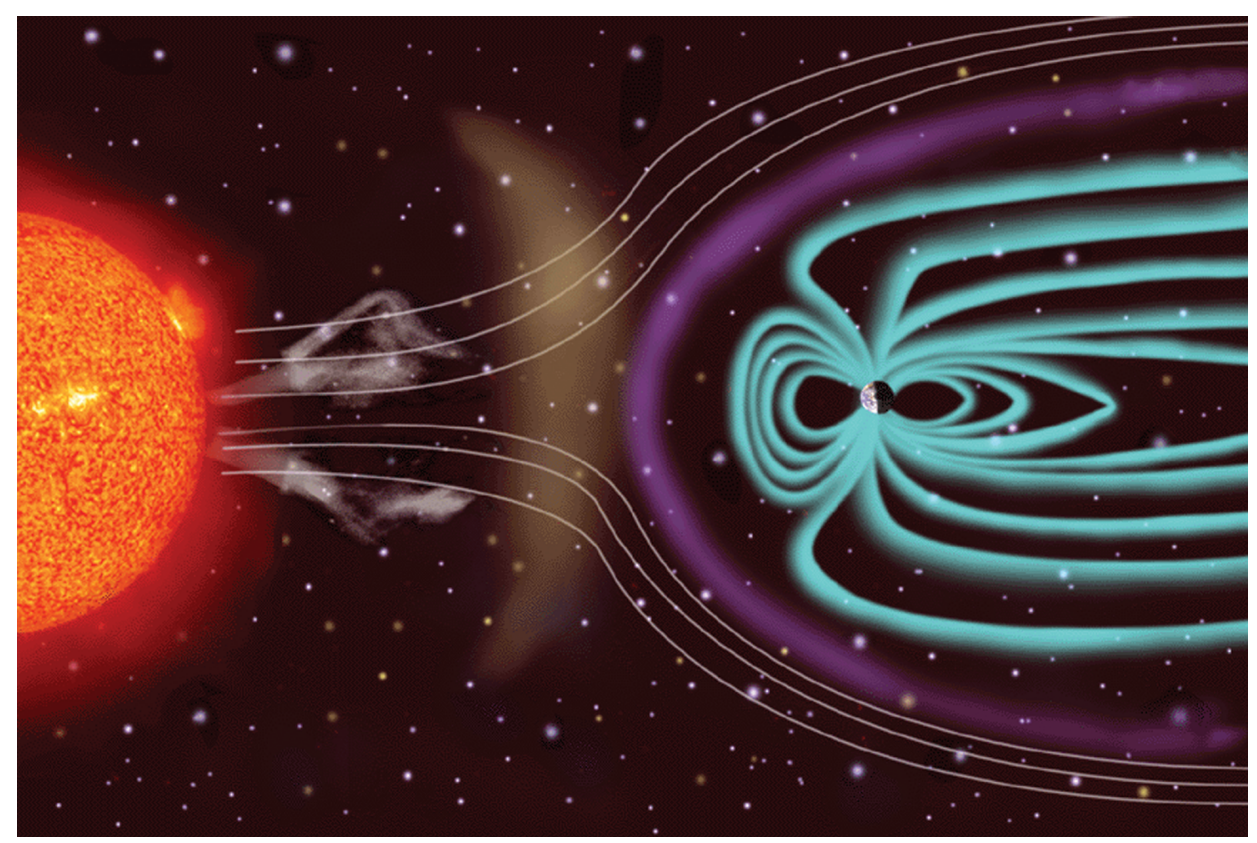

Figure 1. Schematic of the Sun and its interaction with the Earth's magnetic field in space.

\section{The USGS Geomagnetism Program}

The mission of the U.S. Geological Survey's Geomagnetism Program is to monitor the Earth's magnetic field (fig. 2). Using ground-based observatories, the Program provides continuous records of magnetic field variations covering long timescales; disseminates magnetic data to various governmental, academic, and private institutions; and conducts research into the nature of geomagnetic variations for purposes of scientific understanding and hazard mitigation. The Program is an integral part of the U.S. Government's National Space Weather Program (NSWP), which also includes programs in the National Aeronautics and Space Administration (NASA), the Department of Defense (DOD), the National Oceanic and Atmospheric Administration (NOAA), and the National Science Foundation (NSF). The NSWP works to provide timely, accurate, and reliable space weather warnings, observations, specifications, and forecasts, and its work is important for the U.S. economy and national security.

USGS data are used to model and map the global magnetic field, which requires cooperation among the international community of geomagnetism and various satellite programs. The USGS Geomagnetism Program's work with foreign national geomagnetism programs is coordinated through Intermagnet, a worldwide consortium of observatory programs, and the International Association of Geomagnetism and Aeronomy. Regionally, USGS data are used to support aeromagnetic surveys and directional drilling programs for the oil and gas extraction industry. USGS data also are used by the pipeline and electrical power grid industries, as well as for academic studies across a broad range of geophysical sciences. 


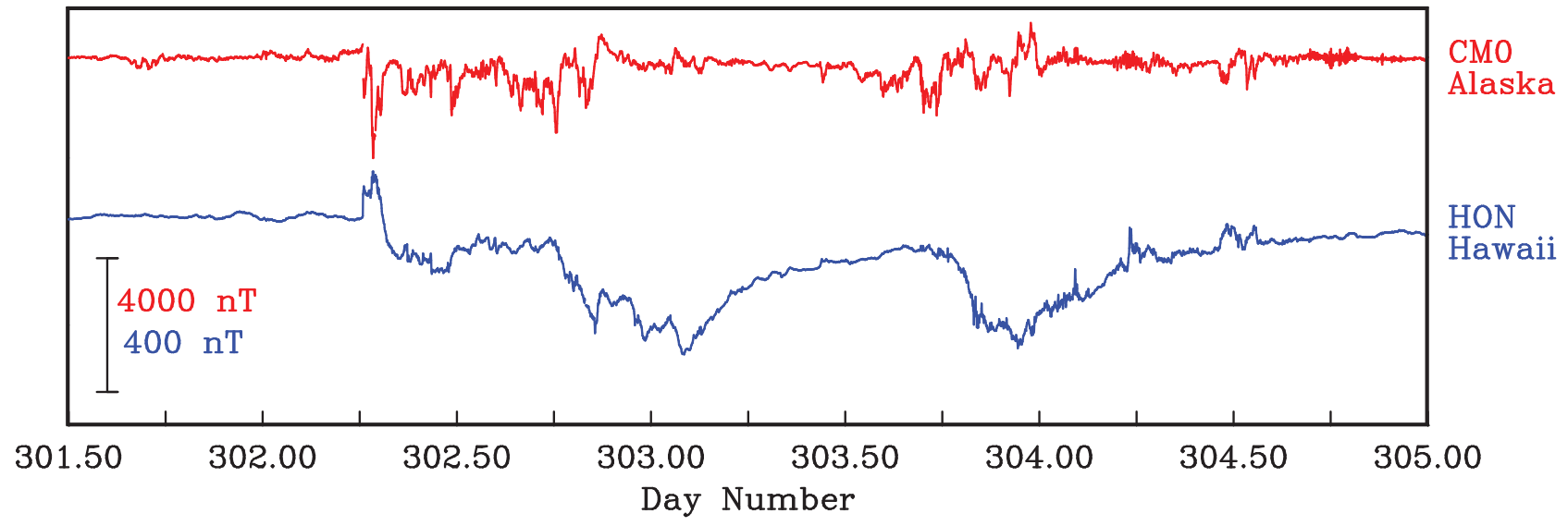

Figure 2. Magnetic data from Honolulu and Alaska recording the Halloween storm of 2003, one of the largest storms ever recorded.

\section{A Long History of Service}

Over the course of its existence, the USGS Geomagnetism Program has evolved in response to changing National needs and organizational changes within various Federal agencies. The Program traces its origins back to 1807 when Congress authorized coastal surveys, including geomagnetic surveys, under the Treasury Department. In the 19th century, magnetic stations were established to help with the development of magnetic maps for the United States and Territories. With the purchase of Alaska, coastal surveys became increasingly important, and so in 1878 the Coast and Geodetic Survey (CGS) was established, with a separate Division of Terrestrial Magnetism established in 1899. The first permanent CGS geomagnetic observatory was established in 1900, and because of the co-location of seismometers on some observatory sites, a fruitful collaboration between Geomagnetism and Seismology began. In 1903, the CGS was transferred to the newly established Department of Commerce and became part of NOAA when that agency was formed in 1970. Soon thereafter, both the Geomagnetism and Seismology Programs in CGS were transferred to the USGS.

\section{The Program Today}

Geomagnetism is headquartered with the USGS Central Region Geologic Hazards Team in Golden, Colorado, which also includes staff supported by the Earthquake Hazards and Landslide Hazards Programs. The engineers and technicians of the Geomagnetism Program are responsible for the collection, transportation, and dissemination of data from 14 observatories (fig. 3) in real time, and the program also has significant data-processing and management capabilities. Accurate measurements are made automatically at each observatory every second. By necessity, everything associated with acquiring and handling the data is technologically elaborate, and the system's many finely tuned components need to be carefully operated and synchronized.

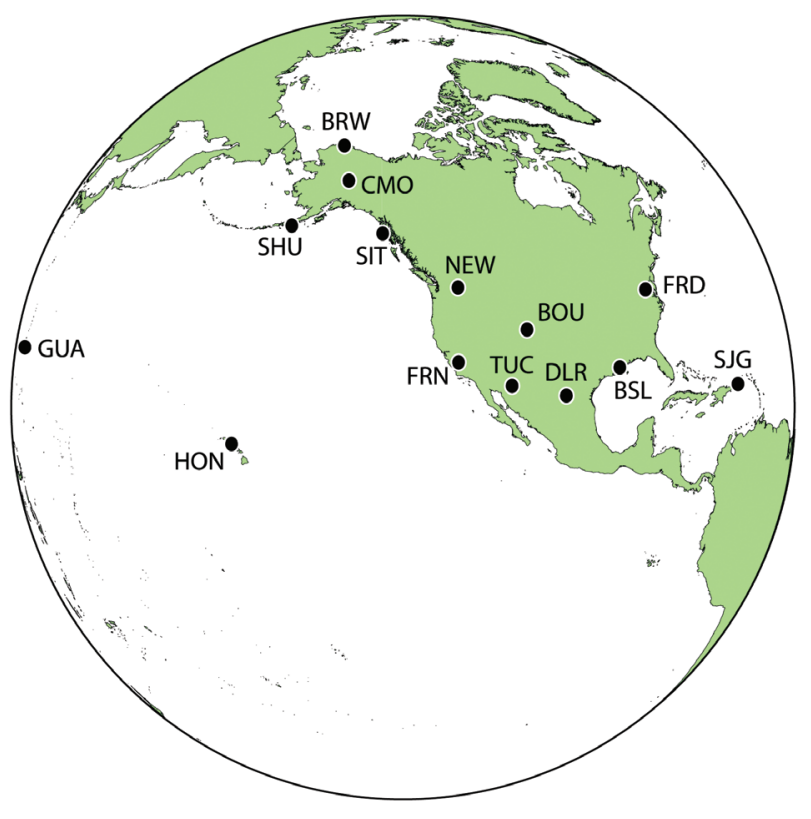

Figure 3. The locations of USGS geomagnetic observatories, with three-letter abbreviations of the observatory names.

\section{Looking to the Future}

In response to the rapidly evolving science of geomagnetism, and the ever more stringent demands of the scientific community, Program staff are constantly upgrading and modernizing the observatory network. In the future, the Geomagnetism Program will monitor the magnetic field at higher frequencies and, when needed, gradually expand the observatory network in collaboration with allied agencies. Program staff are undertaking new scientific research and product development focused on characterizing the complicated behavior of the Earth's magnetic field in space and time, especially during magnetic storms.

\section{Additional Information}

Please visit the National Geomagnetism Program's website, http://geomag.usgs.gov, where you can learn more about the Program and the science of geomagnetism. You can find additional related information at the Intermagnet website, http://www.intermagnet.org.

Jeffrey J. Love

David Applegate

John B. Townshend 